\title{
Utilización de microorganismos para una agricultura sostenible en México: consideraciones y retos
}

\author{
Carlos Iván Cruz-Cárdenas ${ }^{1}$ \\ Lily X. Zelaya Molina ${ }^{2}$ \\ Gabriela Sandoval Cancino ${ }^{3}$ \\ Sergio de los Santos Villalobos ${ }^{4}$ \\ Edith Rojas Anaya ${ }^{1}$ \\ Ismael Fernando Chávez Díaz \\ Santiago Ruíz Ramírez ${ }^{5}$
}

${ }^{1}$ Laboratorio Agrícola Forestal de Semillas Ortodoxas-Centro Nacional de Recursos Genéticos-INIFAP. Boulevard de la Biodiversidad núm. 400, Rancho las Cruces, Tepatitlán de Morelos, Jalisco, México. CP. 47600. Tel. 800 0882222, ext. 84823. (cruz.ivan@ inifap.gob.mx). ${ }^{2}$ Laboratorio de Recursos Genéticos Microbianos (CRNG)-INIFAP. (zelaya.lily@inifap.gob.mx; chavez.fernando@inifap.gob.mx). ${ }^{3}$ Laboratorio Agrícola Forestal de Cultivo in vitro. CRNG-INIFAP. (sandoval.gabriela@inifap.gob.mx). ${ }^{4}$ Instituto Tecnológico de Sonora. 5 de febrero 818 sur, Col. Centro, Ciudad Obregón, Sonora, México. CP. 85000. Tel. 644 4100900, ext. 2124. (sergio.delossantos@itson.edu.mx). ${ }^{5}$ Campo Experimental Centro Altos de Jalisco-INIFAP. Av. Biodiversidad núm. 2470, Rancho las Cruces, Tepatitlán de Morelos, Jalisco, México. CP. 47600. AP. 56. Tel. 800088 2222, ext. 84515. (ruiz.santiago@inifap.gob.mx).

${ }^{\S}$ Autor para correspondencia: ruiz.santiago@inifap.gob.mx.

\section{Resumen}

Numerosas especies vegetales de interés agrícola establecen simbiosis con microorganismos edáficos, tal es el caso de microorganismos promotores del crecimiento vegetal; los cuales proporcionan grandes beneficios debido a que ayudan a disminuir el uso excesivo de fertilizantes y pesticidas utilizados en la producción agrícola. Actualmente, la agricultura mexicana busca alternativas de fertilización amigables con el medio ambiente. Es por ello que las prácticas de agricultura sostenible solamente pueden ser exitosas cuando los productores tienen todos los medios para implementarlas adecuadamente. El presente ensayo aborda temas sobre las consideraciones y retos para el desarrollo de una agricultura sostenible en México, mediante el uso de microorganismos benéficos, y presenta el panorama actual y futuro sobre su uso tomando en cuenta el beneficio para el productor.

Palabras clave: biofertilizantes, microorganismos en el suelo, sostenibilidad.

Recibido: junio de 2021

Aceptado: julio de 2021 
Los niveles de inseguridad alimentaria mundialmente son muy altos debido a la elevada tasa de crecimiento en la población y la limitada innovación para la sostenibilidad de los sistemas de producción agrícola. La importancia de la seguridad alimentaria se da desde un punto de vista socioeconómico, pero también del acceso a los recursos naturales y de la agricultura, y su impacto sobre los recursos de un país (Urquía-Fernández, 2014). Alrededor del mundo existe una amplia demanda de alimentos en países en vías de desarrollo que, aunque puedan contar con los recursos naturales y genéticos para la producción de sus alimentos, la producción no cubre la demanda y además no es sostenible (Sosa, 2017).

Una vía para la mitigación de dichos efectos es conocer su origen, el cual es las formas en las que se producen los alimentos en estas comunidades, dando forma al concepto de agricultura sostenible. Este se enfoca en la producción a largo plazo, tanto de ganadería como de alimentos, impactando lo menos posible en el medio ambiente desde sus factores bióticos como abióticos (Waseem et al., 2020). La acumulación de conocimiento en comunidades o regiones con poco acceso a la tecnología o a prácticas sostenibles permiten que poco a poco los productores sean autosuficientes y tener mayor productividad sin demasiado daño a sus propios recursos, en muchos de los casos, el principal es el racional uso de agua y prácticas de agricultura compatibles con la gestión del uso del suelo, el éxito para la implementación de estas prácticas es la adopción de dichas tecnologías (Fielding et al., 2008).

Desde luego, la implementación de las prácticas de agricultura sostenible solamente puede ser exitosas cuando los productores tienen los medios para poder implementarlas adecuadamente, un ejemplo es lo realizado con la producción sostenible de banana, entre diversas conclusiones presentadas, se identificaron los factores socioeconómicos y psicosociales como aquellos que limitan la implementación de las prácticas sostenibles en la agricultura (Waseem et al., 2020).

De acuerdo con la Academia Nacional de Ciencias de Estados Unidos de América, la producción agrícola (ecológica o convencional) se considera sostenible si y sólo si se producen cantidades adecuadas de alimentos de alta calidad, sin intervenir la base de recursos naturales y el medio ambiente, siendo económicamente viables contribuyendo al bienestar de los agricultores y sus comunidades (Reganold y Wachter, 2016).

\section{Resultados y retos de la agricultura sostenible}

Los métodos de producción agrícola convencionales no están produciendo suficiente alimento para cubrir las necesidades alimenticias de la población nacional creciente (FAO, 2014). Las prácticas de la agricultura convencional están teniendo efectos relacionados con el cambio climático, esto debido al uso de combustibles fósiles, de maquinaria agrícola, fertilizantes y abonos químicos, los cuales generan gases de efecto invernadero, agravando el problema ya que no sólo se pone en riesgo la seguridad alimentaria, sino también se incrementa el daño ambiental (López y Rodríguez, 2014).

México es uno de los países más comprometidos con la mitigación del cambio climático mediante acciones concretas, habiendo firmado el Protocolo de Kioto (1997) y diseñado la estrategia nacional de cambio climático (ENCC) en 2007, en la cual se definen acciones para la agricultura sostenible, tales como la reducción de emisiones del uso de fertilizantes y la labranza de conservación para mantener las reservas de carbono e incrementar la captura (SAGARPA, 2014). 
Las estrategias nacionales han definido acciones concretas a seguir, pero también han surgido otro tipo de herramientas, como la Red Nacional de Desarrollo Rural Sostenible (Rendrus), creada en México en 1996, con el objetivo de que los productores rurales se fortalezcan al identificar, sistematizar e intercambiar experiencias empresariales exitosas, el establecimiento de ésta red permitió una propuesta eficiente para el uso de metodologías para evaluar la sustentabilidad y capacitación a productores con respecto a la agricultura sostenible (PastorPérez et al., 2016).

Además de las estrategias empleadas para implementar la agricultura sostenible, en los últimos años se ha incrementado en uso de microorganismos asociados a los cultivos, para contribuir a la reducción del uso de fertilizantes sintéticos y mitigar la contaminación ambiental causada por éstos (Chávez-Díaz et al., 2020). Se han tenido resultados y avances en cuanto a la agricultura sostenible, aún existen retos para la implementación de estos modelos agrícolas (Bergel, 2020).

Uno de los principales retos está asociado con la economía de los productores, debido a la percepción y balance que hacen respecto al costo/beneficio económico a corto, mediano y largo plazo, ya que todavía existe cierto escepticismo por parte de los usuarios finales, sí bien la producción con técnicas sostenibles representa poca o casi nula inversión a corto o mediano plazo les representa un costo al no poder responder a la demanda del mercado en términos de productividad; sin embargo, en los casos en que consideran la visión a largo plazo, el balance se inclina de nuevo a la agricultura sostenible al considerar el costo por el deterioro del suelo, la pérdida de fertilidad y los servicios multifuncionales de la agricultura (Gerritsen et al., 2012).

A pesar de los avances alcanzados como resultado del trabajo nacional en el ámbito de investigación, difusión y aplicación del conocimiento generado, aún es limitado el avance sobre la regulación y legislación del uso de microorganismos para biofertilización. En general, el principal reto que se debe atender para que se pueda llamar sostenible a la agricultura es asegurar la cantidad necesaria de comida para el futuro, al mismo tiempo que el uso del suelo sea eficiente, se reduzca el impacto al medio ambiente y mejore la economía de los agricultores (López y Rodríguez, 2014).

\section{Importancia de los microorganismos en el suelo}

La actividad microbiana del suelo, y sus beneficios sobre éste, es fuertemente impactada por las prácticas agrícolas intensivas no sostenibles y las condiciones climáticas, a través de modificaciones de las características del suelo a nivel físico, químico y biológico, i.e. temperatura, humedad, salinidad, aireación, estado de óxido-reducción, contenido y composición de los gases en el espacio poroso, biodisponibilidad de los nutrientes, $\mathrm{pH}$ (IbarraVillarreal et al., 2021). De esta manera, el desequilibrio en las comunidades microbianas del suelo desencadena procesos de degradación biológica, reduciendo el rendimiento/calidad de los cultivos al aumentar la vulnerabilidad ante diversos tipos de estrés y limita la capacidad de llevar a cabo sus principales servicios ecosistémicos, tales como: producción de biomasa vegetal, almacenamiento y reciclaje de nutrientes, almacenamiento y filtrado de agua, regulación de clima e inundaciones, mitigación del cambio climático y hábitat para la actividad biológica (Díaz-Rodríguez et al., 2021). 
La bioprospección de los microorganismos que habitan en el suelo representa una herramienta promisoria para el desarrollo de prácticas agrícolas sostenibles enfocadas a satisfacer la demanda de alimentos asociada al incremento poblacional mundial [el cual se proyecta que alcanzará 10 mil millones de habitantes para 2050 y en consecuencia se requerirá un aumento en la producción de alimentos entre 70 y 100\% (FAO, 2017; De los Santos-Villalobos et al., 2018)].

Las comunidades microbianas en los suelos conducen entre 80 y $90 \%$ de los procesos biológicos desarrollados en el suelo (Bajsa et al., 2013), debido a sus múltiples nichos ecológicos, entre los que destacan la mitigación de alteraciones exógenas, promoción de crecimiento vegetal, actividad de biocontrol, ciclaje de nutrientes, producción de biomasa vegetal, estructura y fertilidad del suelo, la degradación de compuestos tóxicos, entre otros (Delgado-Baquerizo et al., 2016).

Entre esta microbiota, se encuentran un conjunto particular denominado microorganismos promotores de crecimiento vegetal (MPCV), los cuales directa o indirectamente favorecen el crecimiento vegetativo, generan tolerancia al estrés abiótico y biótico en la planta, facilitan la nutrición de la planta y antagonizan fitopatógenos en las plantas hospederas. Entre los géneros microbianos más estudiados de este grupo destaca Pseudomonas, Enterobacter, Bacillus, Variovorax, Klebsiella, Burkholderia, Azospirillum, Serratia, Azotobacter y Trichoderma (teleomorfo Hypocrea) (Dohrmann et al., 2013).

Entre las capacidades metabólicas de cepas de MPCV se encuentran la producción de antibióticos peptídicos y metabolitos que protegen a las plantas de posibles ataques de organismos patógenos; además estimulan el sistema inmune de la planta para protegerlas contra infecciones por bacterias, hongos, o nematodos patogénicos (Valenzuela-Ruiz et al., 2020). Por otra parte, diversas cepas de MPCV tienen la capacidad de sintetizar fitohormonas, tanto para regular el crecimiento y desarrollo vegetal como para incrementar la biodisponibilidad de nutrientes en el suelo, permitiendo así una mejor nutrición de la planta (Orozco-Mosqueda y Santoyo, 2020).

Asimismo, los MPCV tiene la capacidad de fijar el nitrógeno atmosférico y solubilizar el fósforo (mecanismos que aportan nutrientes a las plantas, mitigando los eventos de contaminación ambiental causada por la aplicación de fertilizantes sintéticos), así como el secuestro de hierro por sideróforos que previene el desarrollo de fitopatógenos, cuando su crecimiento depende de este elemento (Valenzuela-Aragón et al., 2019; Rojas-Padilla et al., 2020).

De esta manera, los microorganismos benéficos del suelo con aplicación en la agricultura se puede dividir en 1) fitoestimulantes, los cuales potencian la germinación de las semillas, el enraizamiento, y el crecimiento vegetal mediante la producción de reguladores de crecimiento, vitaminas y otras sustancias; 2) mejoradores, los cuales favorecen la estructura del suelo y sus propiedades físico-químicas debido a la formación de agregados, lo cual aumenta su fertilidad; 3) biorremediadores, estos están asociados a la eliminación de agroinsumos sintéticos recalcitrantes y altamente dañinos para el ambiente y salud humana, como pesticidas, herbicidas, entre otros; y 4) biofertilizantes, los cuales tienen la capacidad de proporcionar nutrientes biodisponibles y moléculas bioactivas para el crecimiento y desarrollo incrementado de las plantas, incluyendo el control de fitopatógenos (Joshi et al., 2019). 
Los biofertilizantes, previamente a su uso, deben ser analizados con base en la problemática a atender, su capacidad de colonización del suelo y la planta, la síntesis de compuestos bioactivos de interés y las comunidades microbianas nativas (Chávez-Díaz et al., 2020), ya que su actividad de promoción del crecimiento vegetal se puede centrar a distintos niveles de acción, i.e. actividades metabólicas para la solubilización o mineralización de nutrientes, la biosíntesis de metabolitos benéficos ampliamente estudiados o no descubiertos, la producción de compuestos antagónicos de fitopatógenos (Díaz-Rodríguez et al., 2021).

La importancia de los microorganismos edáficos y su estrecha relación con la agricultura sostenible depende del aprovechamiento de la diversidad metabólica y funcional de los MPCV (Figura 1). Para lo cual, es determinante enfocar esfuerzos y financiamiento para la bioprospección de las comunidades microbianas benéficas y determinar su papel en la red compleja de interacciones físicas, químicas y biológicas ocurridas en el suelo, lo cual conducirá al diseño de estrategias sostenibles para mejorar la fertilidad y salud del suelo, la producción y calidad de los cultivos agrícolas y mitigar el impacto negativo a nivel económico, ambiental y a la salud, del uso de prácticas agrícolas intensivas no sostenibles.

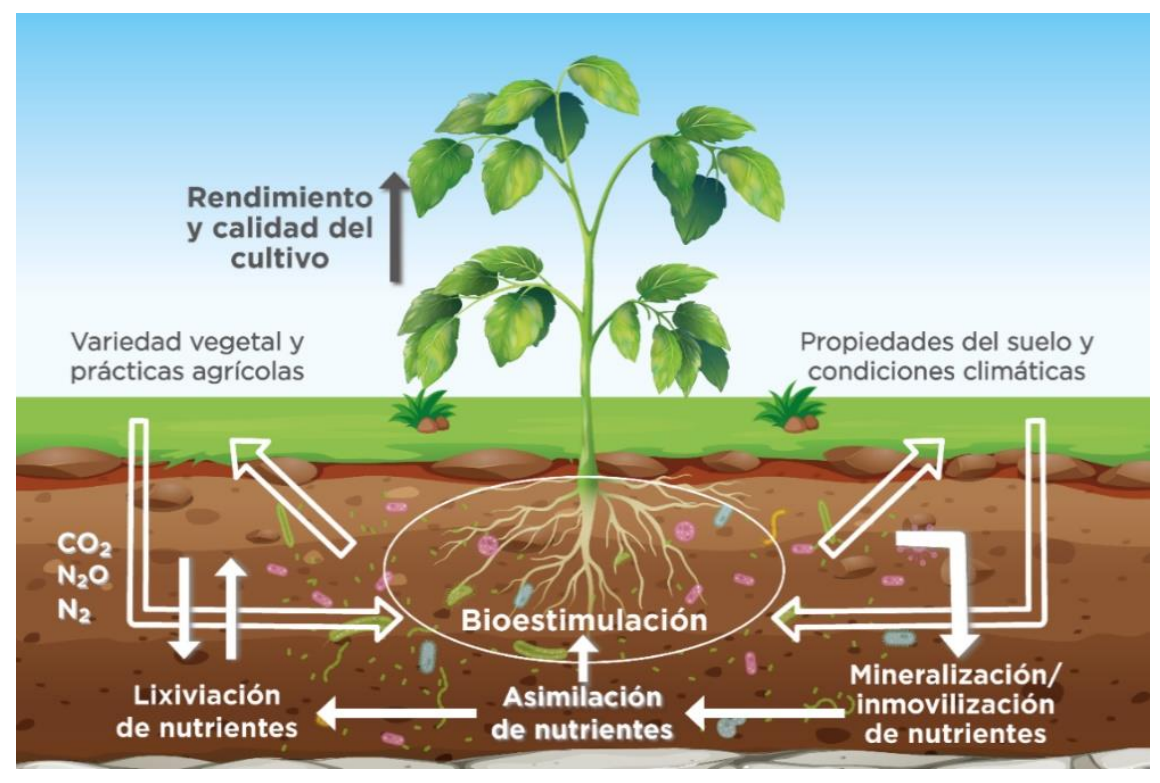

Figura 1. Procesos biológicos regulados por los microorganismos involucrados en la fertilidad del suelo y productividad de las plantas.

\section{Los microorganismos en la agricultura sostenible}

La agricutlura sostenible busca manter en equilibrio a las comunidades microbianas (bacterias, hongos, protozoarios, y virus) asociadas a los cutivos agrícolas, esto es el fitomicrobioma, ya que la diversidad, estabilidad y resilencia del fitomicrobioma son los principales determinantes de la productivia y salud vegetal de un agroecosistema (Basu y Kumar, 2020). Los MPCV, mediante diferentes tipos de relaciones simbióticas que establecen con las plantas, efectuan mecanismos benéficos directos e indirectos para promover el crecimiento de las plantas. 
Con base en el modo de acción que presenten los MPCV, estos se pueden emplear para el desarrollo de bioproductos, como biofertilizantes, fitoestimuladores, biofungicidas o bioplaguicidas (Mamani y Filippone, 2018). Generalmente estos inoculantes microbianos contienen una o varias cepas que presentan diferentes mecanismos de acción, por lo que pueden emplearse en diferentes etapas del ciclo del cultivo; sin embargo, es necesario desarrollar la formulación, producción y los sistemas de administración adecuados para garantizar la supervivencia y el establecimiento efectivo de los MPCV en la planta, así como dar seguimiento del efecto que tienen en la comunidad microbiana previa y la estabilidad de la nueva comunidad generada (Richardson y Simpson, 2011).

En este sentido las técnicas biotecnológicas y moleculares pueden ayudar a comprender mejor el modo de acción de los MPCV para establecer interacciones planta-microrganismo y una aplicación favorable de los MPCV (Khalid et al., 2009), para en un futuro considerar al fitomicrobioma como parte integral de programas de fitomejoramiento (Córdova-Albores et al., 2021).

\section{Desarrollo de un enfoque ecológico para la elección de inoculantes microbianos en México}

En cuanto al origen de los organismos utilizados como biofertilizantes, existen datos que ponen de manifiesto la importancia de la identidad de los simbiontes para incrementar la eficiencia de la asociación y los beneficios para la planta; por ejemplo, la luz (Villegas et al., 2017) y las bajas temperaturas originan cambios en la colonización de las diferentes especies de hongos micorrícicos arbusculares (HMA) (Latef y Chaoxing, 2011).

Por su parte Gavito y Azcón-Aguilar (2012) encontraron que organismos procedentes de zonas frías toleran mejor las bajas temperaturas que los de zonas templadas, ya que presentan variaciones genéticas y plasticidad, lo que les permite adaptarse a esas condiciones ambientales extremas. En habas cultivadas en suelos alcalinos, la inoculación de bacterias fijadoras de nitrógeno y HMA incrementa el crecimiento de la planta medido como biomasa seca total (Abd-Alla et al., 2014). Es sumamente importante tomar en cuenta que a diferencia de los productos agroquímicos, los productos de formulación microbiológica para su buen funcionamiento dependen de las interacciones ecológicas entre las cepas de la formulación, las comunidades microbianas presentes en el suelo y la planta, además de los factores abióticos y climáticos del agroecosistema (Sruthilaxmi y Babu, 2017).

La mayor parte del éxito en el funcionamiento de un producto microbiológico depende del grado del conocimiento ecológico del agroecosistema, en donde el producto se aplicará y de las características de las cepas a utilizar (Compant et al., 2019). Es un hecho que no todos los microorganismos contenidos en estos productos funcionan de la misma manera para todos los cultivos, esto se debe a que los microorganismos han co-evolucionado con las plantas en hábitats particulares, estableciendo interacciones específicas reguladas por los factores agroclimáticos de cada región, a través del tiempo (Pérez-Jaramillo et al., 2018).

Estas propiedades y funciones del suelo son el resultado de una compleja red de interacciones microbianas, las cuales, están moduladas por factores específicos como: 1) la microbiota presente en cada región; 2) las condiciones agroclimáticas específicas del sitio en el que se establece el agroecosistema; 3) el genotipo del cultivo o cultivos establecidos en el agroecosistema; y finalmente 4) el manejo e influencia antropocéntrica sobre el agroecosistema (Compant et al., 2019; Saad et al., 2020). 
Hasta ahora, los formuladores de productos biológicos disponibles en el mercado mexicano se han centrado en algunas cualidades específicas de ciertos microorganismos (Cuadro 1), lo que ha hecho a géneros como Bacillus, Trichoderma, Rhizophagus o Bauberia, atractivos para el productor. No obstante, los microorganismos con los que se formulan los productos biológicos son obtenidos de ambientes diversos y no han coevolucionado con el entorno en el que son liberados, por lo que estos deben adaptarse a las condiciones del medio y a las poblaciones autóctonas del agroecosistema (Basu et al., 2021).

Cuadro 1. Productos biológicos disponibles en el mercado mexicano.

\begin{tabular}{|c|c|c|c|}
\hline Tipo & Desarrollador/fabricante & Producto & Microorganismo \\
\hline $\begin{array}{l}\text { Acondicionar } \\
\text { de suelo }\end{array}$ & zare agrhos & Bioactive az & $\begin{array}{c}\text { Bacillus subtilis, } B . \\
\text { amyloliquefaciens, } B \text {. liqueniformis, } \\
\text { B. megaterium y } B \text {. micoides }\end{array}$ \\
\hline Bioestimulante & Indebio & Pseudofos & Pseudomona fluorescens \\
\hline \multirow[t]{20}{*}{ Biofertilizer } & Agribest & $\begin{array}{l}\text { Nitrobac } \\
\text { plus }\end{array}$ & $\begin{array}{c}\text { Azospirillum brasiliensis, } \\
\text { Azotobacter spp., Bacillus spp. }\end{array}$ \\
\hline & Agrokemyca & & Bacillus subtilis, Pseudomonas \\
\hline & Activa & $\begin{array}{l}\text { Proceveg } \\
\text { plus } \\
\text { Hiper-gram }\end{array}$ & $\begin{array}{c}\text { fluorescens } \\
\text { Gluconacetobacter diazotrophicus, } \\
\text { Azospirillum brasilense, }\end{array}$ \\
\hline & Biofábrica & Hiper-glom & Azotobacter sp. \\
\hline & Siglo xxi & Ctospor & $\begin{array}{c}\text { Glomus intraradices, Pisolithus } \\
\text { tinctorius, Rhizopogon, }\end{array}$ \\
\hline & Biosustenta & Endospor & $\begin{array}{l}\text { amylopogon, } R \text {. bilosuli, } R \text {. } \\
\text { fulvigleba, } R \text {. luteolus, Lacaria }\end{array}$ \\
\hline & Bioqualitum & Fosfonat & $\begin{array}{l}\text { bicolor, L. laccata, Scleroderma } \\
\text { citrini, S. cepa, Trichoderma }\end{array}$ \\
\hline & \multirow[t]{13}{*}{ INIFAP } & Biocomposta & $\begin{array}{c}\text { harzianum, T. reesei, Azospirillum } \\
\text { brasiliense, Azobacter }\end{array}$ \\
\hline & & Azofer plus & chroococcum, Bacillus megaterium, \\
\hline & & Maxifer & Pseudomonas flourescens \\
\hline & & Rhizofer & Rizobacterias fijadoras de \\
\hline & & Micorrizafer & nitrógeno, solubilizadoras de \\
\hline & & Plus & $\begin{array}{l}\text { fósforo y promotoras del } \\
\text { crecimiento, Glomus intraradices, }\end{array}$ \\
\hline & & $\begin{array}{l}\text { Ferbiliq, } \\
\text { Ectomic }\end{array}$ & $\begin{array}{l}\text { G. mosseae, }, \text { G. brasilianum, } G . \\
\text { clarum, } \text {. deserticola }, G .\end{array}$ \\
\hline & & Biosustenta & etunicatum, Gigaspora margarita, \\
\hline & & Azospirillum & Trichoderma harzianum, T. reesei, \\
\hline & & Endomaz & T. viride, Gliocladium virens \\
\hline & & Rhizbio & Glomus intraradices, G. mosseae, \\
\hline & & Rhizbio m+ & \\
\hline & & $\begin{array}{l}\text { Micofert } \\
\text { Micbal }\end{array}$ & $\begin{array}{l}\text { G. brasilianum, G. clarum, } G \text {. } \\
\text { deserticola, G. etunicatum, } \\
\text { Gigaspora margarita }\end{array}$ \\
\hline
\end{tabular}




\begin{tabular}{|c|c|c|c|}
\hline Tipo & Desarrollador/fabricante & Producto & Microorganismo \\
\hline \multirow[t]{4}{*}{ Biofertilizante } & INIFAP & $\begin{array}{l}\text { Biosustenta } \\
\text { Micorrizas } \\
\text { Bactocrop }\end{array}$ & $\begin{array}{l}\text { Azospirillum brasiliense, } \\
\text { Azotobacter chroococcum, } \\
\text { Bacillus megaterium, }\end{array}$ \\
\hline & & & $\begin{array}{c}\text { Pseudomonas fluorescens } \\
\text { Azospirillum brasilense, } \\
\text { hongos micorrízicos arbusculares } \\
\text { Azospirillum brasilense } \\
\text { Azospirillum brasilense } \\
\text { Rhizobium etli, Glomus } \\
\text { Intraradices, } \\
\text { Azospirillum brasilense, } \\
\text { Glomus intraradices, } \\
\text { Ecto micorrizas }\end{array}$ \\
\hline & & & $\begin{array}{c}\text { Azospirillum brasilense } \\
\text { Azospirillum brasilense } \\
\text { Rhizhobium etli }\end{array}$ \\
\hline & & & Bacillus subtilis, B. megaterium \\
\hline Biofortificante & $\begin{array}{l}\text { Biokrone } \\
\text { Grupofagro }\end{array}$ & $\begin{array}{l}\text { Glumix } \\
\text { Irrigation } \\
\text { Glumix } \\
\text { Granulado } \\
\text { Raizorg }\end{array}$ & $\begin{array}{c}\text { Hongos micorrízicos vesículo } \\
\text { arbusculares, Glomus geosporum, } \\
\text { G. fasciculatum, G. constrictum, G. } \\
\text { tortuosum, G. intraradices, } \\
\text { Azospirillum brasilense, } \\
\text { Azotobacter sp., Rhizobium spp., } \\
\text { Bacillus spp. }\end{array}$ \\
\hline \multirow[t]{4}{*}{ OfunBigicida } & $\begin{array}{l}\text { Agrokemyca } \\
\text { altus biopharm }\end{array}$ & $\begin{array}{l}\text { Hiper lisis } \\
\text { Tricho hiper }\end{array}$ & $\begin{array}{c}\text { Bacillus spp., Paenibacillus spp. } \\
\text { Bacillus subtilis, Pseudomonas } \\
\text { fluorescens, B. cereus, B. } \\
\text { megaterium, Lactobacillus sp., }\end{array}$ \\
\hline & $\begin{array}{l}\text { agro\&biotecnia/ibt- } \\
\text { unam } \\
\text { bactiva }\end{array}$ & $\begin{array}{c}\text { Castell } \\
\text { Blitefree }\end{array}$ & $\begin{array}{c}\text { Trichoderma harzianum } \\
\text { Streptomyces spp., Streptomyces }\end{array}$ \\
\hline & $\begin{array}{l}\text { biocampo } \\
\text { biosustenta }\end{array}$ & $\begin{array}{l}\text { Fungifree-ab } \\
\text { Bactiva, } \\
\text { Biosan }\end{array}$ & jofer \\
\hline & biokrone & $\begin{array}{l}\text { Multi-bac } \\
\text { Folisan } \\
\text { Tricsoil }\end{array}$ & $\begin{array}{l}\text { Trichoderma harzianum, } T \text {. reesei, } \\
\text { T. viride, Gliocladium virens, } \\
\text { Bacillus subtilis, B. polymyxa, } B . \\
\text { megaterium, Pseudomonas }\end{array}$ \\
\hline
\end{tabular}




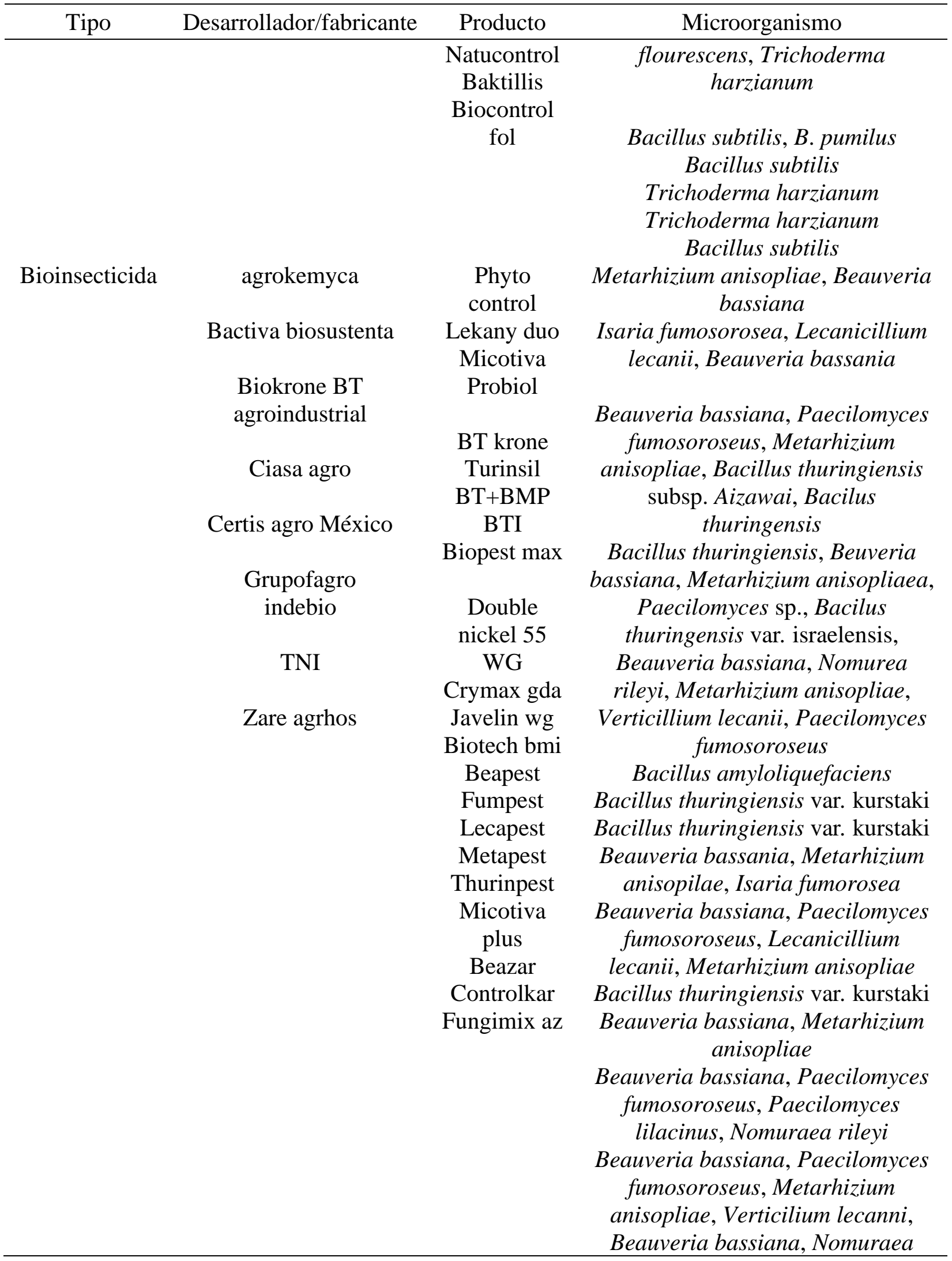




\begin{tabular}{|c|c|c|c|}
\hline Tipo & Desarrollador/fabricante & Producto & Microorganismo \\
\hline $\begin{array}{c}\text { Bioinsecticida- } \\
\text { Bioacaricida }\end{array}$ & Aare agrhos & Thompzar & $\begin{array}{c}\text { rileyi, Metarhizium anisopliae, } \\
\text { Paecilomyces lilacinus, } \\
\text { Paecilomyces fumosoroseus } \\
\text { Bacillus thuringiensis var. Kurstaki, } \\
\text { Metarhizium anisopliae } \\
\text { Heterorhabditis bacteriophora, } \\
\text { Paecilomyces fumoroseus } \\
\text { Hirsutella thompsonii }\end{array}$ \\
\hline Bionematicida & $\begin{array}{c}\text { Agrokemyca } \\
\text { Biosustenta } \\
\text { Grupofagro } \\
\text { Indebio }\end{array}$ & $\begin{array}{l}\text { Hiper nema } \\
\text { Plus } \\
\text { Plcinum } \\
\text { Nemabiol } \\
\text { Plus } \\
\text { Paepest } \\
\text { Pochpest }\end{array}$ & $\begin{array}{c}\text { Paecilomyces lilacinus } \\
\text { Purpureocillium lilacinum } \\
\text { Bacillus subtilis, Trichoderma } \\
\text { harzianum } \\
\text { Paecilomyces lilacinus } \\
\text { Pochonia clamydospora }\end{array}$ \\
\hline $\begin{array}{l}\text { Estimulante } \\
\text { fisiológico }\end{array}$ & Zare agrhos & Biozar & Ascophyllum nodossum \\
\hline $\begin{array}{c}\text { Inoculante } \\
\text { para semilla }\end{array}$ & Zare agrhos & Nitrozar & Rhizobium spp. \\
\hline $\begin{array}{l}\text { Mejorador de } \\
\text { suelo }\end{array}$ & Zare agrhos & $\begin{array}{c}\text { Biofrex } \\
\text { Fijabiol k }\end{array}$ & $\begin{array}{l}\text { Bacillus spp. } \\
\text { Frateuria spp. }\end{array}$ \\
\hline
\end{tabular}

Tomando en cuenta que la utilización de microorganismos en la agricultura obedece a principios ecológicos en los que se busca el buen funcionamiento y equilibrio del agroecosistema es necesaria la correcta elección de un insumo formulado con microorganismos para el campo. Sin embargo, aún se desconoce en gran medida que sucede cuando los microorganismos son liberados en los agroecosistemas, así como las implicaciones ecológicas que podrían suscitarse con las aplicaciones de estos de forma repetida y constante a través del tiempo (Hart et al., 2017).

por lo que siempre al utilizar un producto de formulación microbiológica se debe tomar en cuenta: 1) las cepas utilizadas no supongan un riesgo hacia la salud humana, animal o vegetal, por lo que es recomendable que se avale que tengan una alta expresión de factores de virulencia; 2) las cepas sean nativas del agroecosistema en donde se pretenden aplicar, de tal manera que su actividad se acople fácilmente al medio en el que serán liberadas; y 3) contar con estudios microbiológicos que permita la toma de decisiones en cuanto al uso y manejo de las cepas (Basu et al., 2021).

\section{Avances en política gubernamental con respecto al uso de inoculantes microbianos en México}

Existen temas específicos que permitirían generar avances importantes en materia del uso de estos microrganismos en el desarrollo de una agricultura sostenible en México, los temas se concentran en cuatro aspectos generales: i) actualización en el desarrollo tecnológico de productos microbianos por parte de los especialistas en el campo; ii) impulso en la vinculación de la ciencia con la industria privada; iii) implementación de programas de fomento y apoyo a la investigación en materia de desarrollos microbianos; y iv) establecimiento de una legislación nacional sobre el uso adecuado de estos productos (Salgado-Sánchez, 2015). 
México posee una amplia red de instituciones comprometidas en el desarrollo de productos microbianos y de recursos humanos altamente capacitados en el área, por lo que se han consolidado diversos programas de investigación en materia de microorganismos en muchas entidades académicas a lo largo del país y hoy existe una creciente oferta de especialistas en esta área, muchas de estas instituciones prestan ya servicios a la industria y cada vez es más común el desarrollo de investigación conjunta y de transferencia tecnológica (Chávez-Díaz et al., 2020).

Además, algunos científicos han empezado a generar interés en las 'spin off's', empresas que se derivan de desarrollos tecnológicos realizados en universidades o centros de investigación, con una licencia de uso de dicha tecnología, ya que ofrecen a la industria productos y servicios muy especializados que podrían ser de gran ayuda al desarrollo sostenible de nuestro país (Hernández et al., 2017). El aún limitado número de leyes, normas y reglamentos que regulen el desarrollo de tecnologías basadas en microorganismos y su uso en la agricultura sostenible, implica un rezago en el crecimiento de la agricultura del país (Sabourin et al., 2017).

En México, existen esfuerzos en materia regulatoria como la Norma Oficial Mexicana NOM-077FITO-2000. Estudios de efectividad biológica en insumos de nutrición vegetal de uso agrícola y su 'dictamen técnico', norma oficial en la que se establecen los requisitos y especificaciones para la realización de estudios de efectividad biológica de los insumos de nutrición vegetal; sin embargo, es determinante la generación o actualización de normas para aquellas tecnologías basadas en microorganismos ya que se enfoca principalmente en nutrición química más no biológica y la protección de derechos de autor aún no ha sido abordado de una manera diligente.

Por lo cual, seguir con las estrategias para solventar los retos y consideraciones para fomentar el desarrollo sostenible mediante la utilización de los microorganismos permitirá fortalecer al sector primario como a diversos sectores productivos existentes, generando una oferta de mayor valor agregado a los productos agrícolas. Asimismo, puede contribuir a resolver muchas necesidades sociales que se identifican en México y a nivel mundial, como: abasto de alimentos, cuidado de los recursos naturales, del medio ambiente y mejorar la calidad de vida, por mencionar algunos.

\section{Perspectivas para la divulgación del uso de los inoculantes microbianos en México}

En México, el uso de microorganismos en la agricultura ha avanzado a través de las últimas décadas; lograr que estos avances permeen a los productores por medio de la divulgación es el mejor camino para que la ciencia sea cada vez más vista como una herramienta necesaria en el desarrollo sostenible de nuestro país.

En este sentido, en el Instituto Nacional de Investigaciones Forestales Agrícolas y Pecuarias (INIFAP), se han desarrollado y consolidado diversos esfuerzos en materia de divulgación del uso de los microorganismos en la agricultura en México, tal como el AgroEvento 2020 'productos biológicos, una herramienta para potencializar el campo mexicano' (AgroEvento, 2020), organizado por el Centro Nacional de Recursos Genéticos (CNGR) del INIFAP y el Instituto Tecnológico de Sonora, dicho evento tuvo como objetivo reunir a académicos, científicos, estudiantes, técnicos, asesores, capacitadores, comercializadores, productores de insumos agrícolas y agricultores a compartir sus experiencias, conocimientos y perspectivas referentes al uso de productos biológicos, $\mathrm{u}$ otras agrobiotecnologías para la innovación sostenible como alternativa a la producción agrícola convencional en México. 
Este tipo de eventos permite a investigadores mexicanos involucrados en el desarrollo e implementación de tecnologías basadas en microorganismos que propicien la conservación y el aprovechamiento de la biodiversidad nacional, con este tipo de acciones se informa a productores y personas del sector agropecuario sobre los avances en materia de aislamiento, identificación, y caracterización de microorganismos benéficos para los cultivos, diseño, aplicación, manejo y formulación de productos de formulación biológica para el campo. Esfuerzos como éste se deben de promover para lograr que los productores tengan acceso a la información y puedan decidir cómo aprovechar los avances en materia de uso de microorganismos en la agricultura con el soporte de la investigación científica.

\section{Conclusiones}

Para asegurar el correcto uso de los microorganismos en la agricultura sostenible, se debe asegurar la rentabilidad de estos para el agricultor, pero que a su vez sea amigable con el ambiente. Esto se logra llevando a cabo prácticas agronómicas donde exista la utilización de inoculantes microbianos, de comprobada actividad y pureza, que le aseguran al agricultor un número específico adecuado por especie, que le dan garantía de calidad y por ende confianza.

En el país existen problemáticas como la recuperación de suelos y la mejora en la productividad de los cultivos donde el uso de los microorganismos es una alternativa promisoria. Esta solución es posible si se planea con criterios de sostenibilidad, que involucren soluciones microbiológicas, con nuevos productos de menor o nulo impacto sobre el ambiente.

México tiene la responsabilidad de avanzar en la obtención de inoculantes microbianos seguros para el agricultor, capaces de lograr incrementos en el rendimiento de los cultivos y que además sean seguros para el agroecosistema. La utilización adecuada de los microorganismos en la agricultura sostenible en México se ha descuidado debido a la falta de regulación y el uso indiscriminado de los mismos, por lo que es necesario conectar el uso de estos inoculantes microbianos con la mejora en la producción de alimentos, mediante la toma de acciones para utilizar, restaurar y preservar a los microorganismos como recurso genético.

\section{Agradecimientos}

A la Dra. Edith Rojas Anaya. Laboratorio de recursos microbianos, Centro Nacional de Recursos Genéticos-INIFAP, por su colaboración y aportación en el presente trabajo.

\section{Literatura citada}

Abd-Alla, M. H.; El-Enany, A. W. E.; Nafady, N. A.; Khalaf, D. M. and Morsy, F. M. 2014. Synergistic interaction of rhizobium leguminosarum BV. Viciae and arbuscular mycorrhizal fungi as a plant growth promoting biofertilizers for faba bean (Vicia faba L.) in alkaline soil. Microbiol. Res. 169(1):49-58. https://doi.org/10.1016/j.micres. 2013.07.007.

Agroevento. 2020. http://cmcnrg.inifap.gob.mx. 
Bajsa, N.; Morel, M. A.; Braña, V. and Castro-Sowinski, S. 2013. The effect of agricultural practices on resident soil microbial communities: focus on biocontrol and biofertilization. Mol. Microbial Ecol. Rhizosphere. 2:687-700.

Basu, A.; Prasad, P.; Das, S. N; Kalam, S.; Sayyed, R. Z; Reddy, M. S. and Enshasy, H. E. 2021. Plant growth promoting rhizobacteria (PGPR) as green bioinoculants: recent developments, constraints, and prospects. Sustainabiliy. 13(3):1140. https://doi.org/10.3390/ su13031140.

Basu, S. and Kumar, G. 2020. Stress signalling in the phytomicrobiome: breadth and potential. In: kumar, M.; Kumar, V. and Prasad, R. (Ed.). Phyto-microbiome in stress regulation. Springer, Singapore. 245-268 pp. https://doi.org/10.1007/978-981-15-2576-6_12.

Bergel, S. D. 2020. Desarrollo sustentable y medio ambiente: la perspectiva latinoamericana. Alegatos. 1(24):196-221.

Chávez-Díaz, I. F.; Zelaya-Molina, L. X.; Cruz-Cárdenas, C. I.; Rojas-Anaya, E.; Ruíz-Ramírez, S. y Santos-Villalobos, S. 2020. Consideraciones sobre el uso de biofertilizantes como alternativa agro-biotecnológica sostenible para la seguridad alimentaria en México. Rev. Mex. Cienc. Agríc. 11(6):1423-1436.

Compant, S.; Samad, A; Faist, H. and Sessitsch, A. 2019. A review on the plant microbiome: Ecology, functions, and emerging trends in microbial application. J. Adv. Res. 19:29-37. https://doi.org/10.1016/j.jare.2019.03.004.

Córdova, A.; Liliana, C.; Zelaya, M.; Lily, X.; Ávila, A.; Norma, V. R.; Valeria, C. M.; Nelly, E.; Parra, C.; Fannie, I.; Burgos, C.; Yamily, Y.; Chávez, D.; Ismael, F.; Fajardo, F.; Marja, L.; Santos, V. and Sergio, D. 2021. Omics sciences potential on bioprospecting of biological control microbial agents: the case of the Mexican agro-biotechnology. Rev. Mex. Fitopatol. 39(1):147-184.

De los Santos, V. S.; Parra-Cota, F. I.; Herrera-Sepúlveda, A.; Valenzuela-Aragón, B. y EstradaMora, J. C. 2018. Colmena: colección de microorganismos edáficos y endófitos nativos, para contribuir a la seguridad alimentaria nacional. Rev. Mex. Cienc. Agríc. 9(1):191-202.

Delgado-Baquerizo, M.; Maestre, F. T.; Reich, P. B.; Jeffries, T. C.; Gaitan, J. J.; Encinar, D.; Berdugo, M.; Campbell, C. D. and Singh, B. K. 2016. Microbial diversity drives multifunctionality in terrestrial ecosystems. Nat. Comm. 7(1):1-8.

Díaz-Rodríguez, A. M.; Salcedo, L. A.; Felix, C. M.; Parra-Cota, F. I.; Santoyo, G.; Puente, M. L.; Bhattacharya, D.; Mukherjee, J. and De los Santos, V. S. 2021. The current and future role of microbial culture collections in food security worldwide. Front. Sustain. Food Syst. 4:614739.

Dohrmann, A. B.; Küting, M.; Jünemann, S.; Jaenicke, S.; Schlüter, A. and Tebbe, C. C. 2013. Importance of rare taxa for bacterial diversity in the rhizosphere of Bt-and conventional maize varieties. The ISME journal. 7(1):37-49.

Fielding, K. S.; Terry, D. J.; Masser, B. and Hogg, M. A. 2008. Integrating social identity theory and the theory of plannedbehaviour to explain decisions to engage in sustainable agricultural practices. Br. J. Soc. Psychol. 47(1):23-48.

FAO. 2017. Food and Agriculture Organization of the United Nations. Towards zero hunger and sustainability. The FAO multipartner programme support mechanism. http://www.fao.org/ documents/card/es/c/fa6a801c-5bd4-4522-a2ff-bfbef1e56529.

Gavito, M. E. and Azcón-Aguilar, C. 2012. Temperature stress in arbuscular mycorrhizal fungi: a test for adaptation to soil temperature in three isolates of Funneliformis mosseae from different climates. Agr. Food Sci. 21(1):2-11. https://doi.org/10.23986/afsci.4994. 
Hernández, R. V. R.; Escandón, J. M. S.; Mendoza, A. L. and Izaguirre, J. A. H. 2017. La tecnología: una herramienta de apoyo para pymes y emprendedores desde el entorno universitario. Ciencia ergo-sum, Rev. Cient. Multidisciplinaria de Prospectiva. 24(1):75-82.

Ibarra-Villarreal, A.; Gándara-Ledezma, A.; Godoy-Flores, A.; Díaz-Rodríguez, A. M.; ParraCota, F. I. and De los Santos, V. S. 2021. Salt-tolerant bacillus species as a promising strategy to mitigate the salinity stress in wheat (Triticum turgidum subsp. durum). J. Arid Environ. 186:104399.

Joshi, H.; Somduttand, C. P. and Mundra, S. L. 2019. Role of effective microorganisms (EM) in sustainable agriculture. Inter. J. Curr. Microbiol. Appl. Sci. 8(3):172-181.

Khalid, A.; Arshad, M.; Shaharoona, B. and Mahmood, T. 2009. Plant growth promoting rhizobacteria and sustainable agriculture. In: Khan, M. S.; Zaidi, A. and Musarrat, J. (Ed.). Microbial strategies for crop improvement. Springer, Berlin, Heidelberg. 133-160 p. https://doi.org/10.1007/978-3-642-01979-1_7.

Latef, A. A. H. A. and Chaoxing, H. 2011. Arbuscular mycorrhizal influence on growth, photosynthetic pigments, osmotic adjustment and oxidative stress in tomato plants subjected to low temperature stress. Acta Physiologiae Plantarum. 33:1217-1225. https://doi.org/10.1007/s11738-010-0650-3.

López, J. y Rodríguez, R. 2014. Agricultura biointensiva: método de agricultura sostenible para los microagricultores de México. In: XVIII Congreso Internacional de Investigación en Ciencias Administrativas. 2434-2455 p.

Orozco-Mosqueda, M. y Santoyo, G. 2020. Bacterias promotoras del crecimiento vegetal: aspectos básicos y aplicaciones para una agricultura sostenible. Primera (Ed.). Fontamara. Ciudad de México, México. 240 p.

Pastor-Pérez, M. D. P.; Ramos-Ávila, A. E. y Santa María-Torres, A. 2016. Evaluación de la sustentabilidad: una reflexión a partir del caso de la red nacional de desarrollo rural sostenible (México). Entreciencias: diálogos en la sociedad del conocimiento. 4(9):61-72.

Pérez-Jaramillo, J. E.; Carrión, V. J.; Hollander, M. and Raaijmakers, J. M. 2018. The wild side of plant microbiomes. Microbiome. 6:143. https://doi.org/10.1186/s40168-018-0519-z.

Reganold, P. J. and Wachter, J. M. 2016. Organic agriculture in the twenty-first century. Nature Plants. 2:1-8.

Richardson, A. E. and Simpson, R. J. 2011. Soil microorganisms mediating phosphorus availability update on microbial phosphorus. Plant Physiol. 156(3):989-996. https://doi.org/10.1104/pp.111.175448.

Rojas-Padilla, J.; Chaparro-Encinas, L. A.; Robles-Montoya, R. I. y De los Santos, V. S. 2020. Promoción de crecimiento en trigo (Triticum turgidum L. subsp. Durum) por la coinoculación de cepas nativas de Bacillus aisladas del valle del Yaqui, México. Nova Scientia. 12(24):1-27.

Saad, M. M.; Eida, A. A. and Hirt, H. 2020. Tailoring plant-associated microbial inoculants in agriculture: a roadmap for successful application. J. Exp. Bot. 71(13):3878-3901. doi:10.1093/jxb/eraa111.

Sabourin, E. P.; Patrouilleau, M. M.; Le-Coq, J. F.; Vásquez, L. y Niederle, P. A. 2017. Políticas públicas a favor de la agroecología en América Latina y el Caribe. Red políticas públicas en América Latina y el Caribe (Red PP-LA). 213 p. 
SAGARPA. 2014. Secretaría de Agricultura, Ganadería, Desarrollo Rural, Pesca y Alimentación (SAGARPA). México: el sector agropecuario ante el desafío del cambio climático. Organización de las Naciones Unidas para la Alimentación y la Agricultura (FAO).

Salgado-Sánchez, R. 2015. Agricultura sostenible y sus posibilidades en relación con consumidores urbanos. Estudios Sociales. 23(45):113-140.

Sosa, B. A. 2017. La disponibilidad de alimentos en México: un análisis de la producción agrícola de 35 años y su proyección para 2050. Papeles de Población. 23(93):207-230.

Sruthilaxmi, C. B. and Babu, S. 2017. Microbial bio-inoculants in Indian agriculture: ecological perspectives for a more optimized use. Agri. Ecosyst. Agroecosyst. Environ. 242(1):2325. http://dx.doi.org/10.1016/j.agee.2017.03.019.

Urquía-Fernández, N. 2014. La seguridad alimentaria en México. Salud Pública de México. 56(1):92-98.

Valenzuela-Aragón, B.; Parra-Cota, F. I.; Santoyo, G.; Arellano-Wattenbarger, G. L. and De los Santos, V. S. 2019. Plant-assisted selection: a promising alternative for in vivo identification of wheat (Triticum turgidum L. subsp. Durum) growth promoting bacteria. Plant and Soil. 435(1-2):367-384.

Valenzuela-Ruiz, V.; Gálvez-Gamboa, G. T.; Villa-Rodríguez, E.; Parra-Cota F. I.; Gustavo, S. y De los Santos, V. S. 2020. Lipopéptidos producidos por agentes de control biológico del género Bacillus: revisión de herramientas analíticas utilizadas para su estudio. Rev. Mex. Cienc. Agríc. 11(2):419-432.

Villegas-Olivera, J. A.; J. Pérez-Moreno, G.; Mata, J. J.; Almaraz-Suárez, D.; Ojeda, T. and. Espinosa-Hernández, V. 2017. Type of light and formation of basidiomata of two species of edible ectomycorrhizal mushrooms associated with neo-tropical pines and the description of basidiomata development. Rev. Fitotec. Mex. 40(4):405-413.

Waseem, R.; Gershom, E. M. G. E.; Waseem, F.; Khan, H.; Ghulam, M.; Panhwar, M. G. and Shi, Y. 2020. Adoption of sustainable agriculture practices in banana farm production: a study from the sindh region of pakistan. Intr. J. Env. Res. Publ. Health. 17:3714-3728. 